\title{
Antiproliferative Activity of Olive Oil Phenolics against Human Melanoma Cells
}

\author{
A.L.M. Batista de Carvalho ${ }^{\mathrm{a}}$, F. Caselli ${ }^{\mathrm{a}}$, V. Rodrigues ${ }^{\mathrm{a}, \mathrm{b}}$, F. Paiva-Martins ${ }^{\mathrm{b}, \mathrm{c}}$ and \\ M.P.M. Marques ${ }^{\mathrm{a}, \mathrm{d}, *}$
}

${ }^{a}$ Unidade de I\&D Quimica-Física Molecular, Department of Chemistry, University of Coimbra, Coimbra, Portugal; ${ }^{b}$ Faculty of Science, University of Porto, Porto, Portugal; ${ }^{c}$ REQUIMTE-LAQV, Porto, Portugal; ${ }^{d}$ Department of Life Sciences, Faculty of Science and Technology, University of Coimbra, Coimbra, Portugal

\begin{abstract}
Background: Virgin olive oil, the main source of lipids in a mediterranean diet, is broadly recognised to possess health-beneficial features, namely a protective role against cancer. It comprises several phenolic compounds, the major ones being tyrosol ( $p$-hydroxyphenylethanol), hydroxytyrosol (3,4-dihydroxyphenylethanol), lignans and secoiridoids, namely oleuropein (OP) and the oleuropein aglycones 3,4-(dihydroxyphenyl) ethanol elenolic acid ester and 3,4-(dihydroxyphenyl)ethanol elenolic acid di-aldehyde.

Objective: The goal of this work is the in vitro evaluation of the anti-proliferative activity against human amelanotic melanoma (C32 cell line) for hydroxytyrosol and the most important secoiridoids of olive oil.

The effect of hydroxytyrosol on non-neoplastic cells (BJ fibroblass) was also investigated.

Methods: Inhibition of cell proliferation was assessed by the Sulforhodamine colorimetric method, in both neoplastic and non-tumorigenic cells.

Results: 3,4-(Dihydroxyphenyl)ethanol elenolic acid di-aldehyde and 3,4-(dihydroxyphenyl)ethanol elenolic acid ester secoiridoid aglycones were found to display growth-inhibiting activity (at $c a .100 \mu \mathrm{M})$, as opposed to oleuropein that elicited a strong protective effect at all concentrations (100 to $1000 \mu \mathrm{M}$ ). 3,4-Dihydroxyphenylethanol evidenced a dual effect (strongly dose-dependent) - cytoprotective for lower dosages and cytotoxic at high concentrations.

Conclusions: Attending to the recognised structural dependence of the biological activity of phenolic derivatives, the previously gathered conformational data on the olive oil constituents presently investigated assisted the interpretation of their biological properties. This type of studies, coupling structural characterisation to biological assessment, allows the establishment of reliable structure activity relationships for polyphenolic compounds, ruling their cytoprotective $v s$ cytotoxic activity and therefore their potential use as natural-based pharmacological agents.
\end{abstract}

\section{A R T I C L E H IS T O R Y}

Received: November 16, 2016 Revised: January 02,2017 Accepted: January 06, 2017 DOI: $10.2174 / 15701808146661701101456$ 58

Keywords: Virgin olive oil, hydroxytyrosol, secoiridoid polyphenols, anticancer, melanoma, fibroblasts.

\section{INTRODUCTION}

Virgin olive oil (VOO), the main source of lipids in a mediterranean diet, is broadly recognised to possess healthbeneficial features, namely a protective role against cancer [1-4]. It comprises several phenolic compounds, the major ones being hydroxytyrosol (3,4-dihydroxyphenylethanol, 3,4-DHPEA), tyrosol ( $p$-hydroxyphenylethanol, $p$-HPEA), lignans (such as (1)-pinoresinol and (1)-1-acetoxy-pinoresinol) and secoiridoids, namely oleuropein (OP) and the oleuropein aglycones 3,4-(dihydroxyphenyl) ethanol elenolic acid ester

*Address correspondence to this author at the Departamento de Ciências da Vida, Faculdade de Ciências e Tecnologia,Universidade de Coimbra, Calçada Martim de Freitas, 3000-456 Coimbra, Portugal; Tel/Fax: +351 239 826 541; E-mail: pmc@ci.uc.pt
(3,4-DHPEA-EA) and 3,4-(dihydroxyphenyl)ethanol elenolic acid di-aldehyde (3,4-DHPEA-EDA) (Fig. 1).

Epidemiological evidence, in vitro and animal studies indicates a protective role of olive oil phenolics (OVPs) against cancer [5-10], as well as an inhibitory effect regarding different stages of carcinogenesis, including angiogenesis and metastasis. Actually, a clear association between the consumption of olive oil (namely in a Mediterranean diet) and a reduced risk of cancer has been reported, namely regarding breast $[11,12]$, prostate [13], lung [14], larynx [15], ovary [16], colon [17] and bladder cancer [9]. Also, a study by Owen and coworkers suggested that a fraction of the ingested OVPs may reach the colon and confer chemopreventive effects against colorectal cancer [1]. In addition, olive oil phenolic extracts have recently been shown to exert 


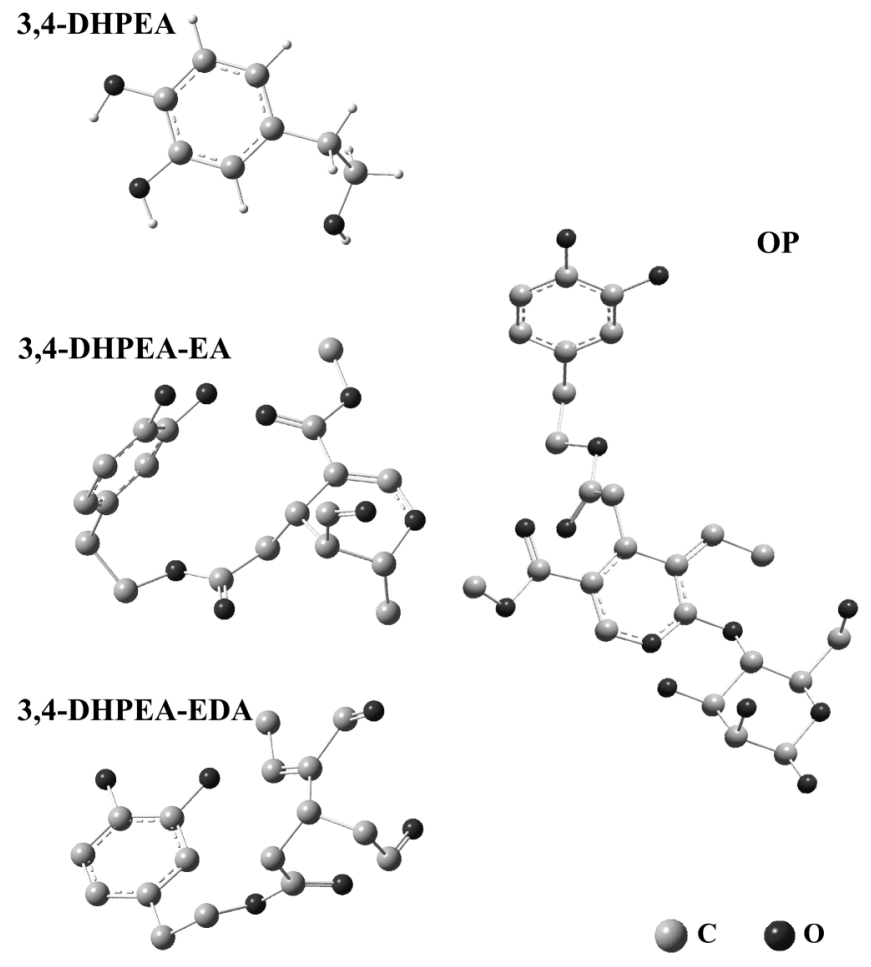

Fig. (1). Schematic representation of the most stable geometries calculated [22] for the olive oil polyphenols (OVPs) studied in the present work: 3,4-DHPEA, OP, 3,4-DHPEA-EA and 3,4-DHPEAEDA. (Except for the smaller molecule - 3,4-DHPEA - the hydrogen atoms are not shown, for clarity sake).

an anti-proliferative effect towards human promyelocytic leukemia cells (HL60) [18-20], as well an inhibitory action towards cell attachment and invasion in HT115 human colon carcinoma [21].

Vissers and colleagues [23] suggested that an important step in the metabolism of oleuropein and oleuropein aglycones is their hydrolysis into either hydroxytyrosol or tyrosol. As a consequence, it has been observed that 3,4DHPEA rises early after virgin olive oil ingestion, reaching a peak after $c a .1 \mathrm{~h}$ in plasma and $0-2 \mathrm{~h}$ in urine [24-26], undergoing an extensive intestinal/hepatic metabolism [24, 27-30]. Hydroxytyrosol was reported to exert a marked antioxidant activity and prevent metal ion catalysed production of reactive oxygen species in bulk oils [31-34], and was also found to avoid LDL oxidation both in vitro and ex vivo [35-37], and to reduce colon cancer growth [10]. In addition, there are some studies evidencing a significant antioxidant effect from this compound towards cultured cancer cells, from epithelial colorectal [38] and melanoma [39] to prostate adenocarcinoma [40].

The biological activity of this type of olive oil phenolic compounds towards cancer is mainly due to their high antioxidant capacity $[25,41]$, through free radical scavenging, metal chelation [42] and modulation of cell signalling pathways and gene expression [43]. However, there are other mechanisms likely to be associated to this antitumour activity, namely at the invasion and metastasis level, possibly involving prevention of attachment of cancer cells to extracellular matrices [21]. The secoiridoid aglycones 3,4-
DHPEA-EA and 3,4-DHPEA-EDA, which are present in olive oil at higher concentrations than hydroxytyrosol, are thought to be the main responsible for the high antioxidant capacity of this food product.

However, despite the widely accepted chemopreventive and antineoplastic properties of olive oil phenolics, the specific components responsible for them and the mechanisms underlying their action remain largely unknown. Actually, very few studies have been reported to this date on the molecular basis of this health-beneficial activity of OVPs. It is generally accepted that the antioxidant properties of these phenolic constituents are conferred by their ortho-diphenolic (catecholic) moieties [44, 45] (Fig. 1). These structural and conformational characteristics, which determine biological activity, namely chemoprevention against oxidative-induced pathologies, have been previously studied by the authors through Raman spectroscopy coupled to DFT calculations [22].

Although melanoma represents only $4-5 \%$ of all skin cancers, it contributes to $85 \%$ of skin cancer deaths, with rising rates mainly due to sunlight overexposure (UV radiation). Amelanotic melanoma is the most dangerous form of skin cancer, with a very poor prognosis and short survival periods. Moreover, metastasis from malignant melanoma occurs mainly in lung, which drastically increases the mortality attributed to this kind of neoplasia.

The present work reports the in vitro evaluation of the anti-proliferative activity against human amelanotic melanoma (C32 cell line) for 3,4-DHPEA and the most important secoiridoids present in olive oil - OP, 3,4-DHPEA-EDA and 3,4-DHPEA-EA. Inhibition of cell proliferation was assessed by the Sulforhodamine (SRB) colorimetric method, that measures cell density. The effect of 3,4-DHPEA on nonneoplastic cells (BJ fibroblast cell line) was also investigated.

\section{MATERIALS AND METHODS}

\section{Chemicals}

Antibiotics (penicillin-streptomycin 100x solution), dimethyl sulfoxide (DMSO), Dulbecco's Modified Eagle's Medium - high glucose (DMEM-HG, $4500 \mathrm{mg} / \mathrm{L}$ glucose), ethylenediaminetetraacetic acid (EDTA, disodium salt, dihydrate), phosphate buffered saline (PBS), Sulforhodamine B (SRB, monosodium salt, $0.5 \%(w / v)$ solution), TRIS (tris(hydroxymethyl)aminoethane), Trypan blue $(0.04 \%$ $(w / v)$ solution), trypsin-EDTA (1x), and inorganic salts and acids (of analytical grade) were purchased from SigmaAldrich Chemical S.A. (Sintra, Portugal). Fetal bovine serum (FBS) was obtained from Gibco-Life Technologies (Porto, Portugal).

\section{Phenolic Compounds}

Hydroxytyrosol (3,4-DHPEA) was synthesised from 3,4dihydroxyphenylacetic acid (Sigma-Aldrich, S. A. Sintra, Portugal) according to Baraldi et al. [46]. Oleuropein and 3,4-DHPEA-EDA were extracted from olive leaves, according to the procedures of Gariboldi [47] and PaivaMartins [48], respectively. The aglycone 3,4-DHPEA-EA was obtained from oleuropein by an enzymatic reaction 
(A)

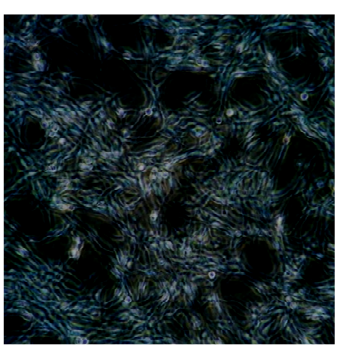

(B)

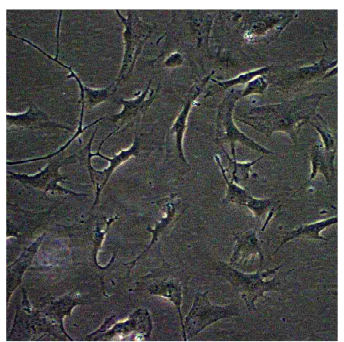

Fig. (2). Microscopic images of the amelanotic melanoma C32 (A) and fibroblasts BJ (B) human cell lines. (Digital images were acquired with a camera coupled to an inverted microscope (Olympus, Portugal) at a 20x magnification).

using $\beta$-glycosidase, following the method reported by Limirioli et al [49].

Solutions of these phenolic compounds (3,4-DHPEA, OP, 3,4-DHPEA-EDA and 3,4-DHPEA-EA) were prepared in DMSO, for a concentration range between $1.0 \times 10^{-4} \mathrm{M}$ $(100 \mu \mathrm{M})$ and $1.0 \times 10^{-3} \mathrm{M}(1000 \mu \mathrm{M})$. Fresh solutions were prepared monthly and kept in the dark, at $5^{\circ} \mathrm{C}$, in order to prevent oxidation. These solutions were sterilised by filtration before being added to the cell cultures.

\section{Biological Assays-Cell Growth Inhibition Evaluation}

The human cell lines were purchased from the American Type Culture Collection (ATCC): amelanotic melanoma (C32) and non-neoplastic male newborn fibroblasts (BJ) (Fig. 2).

C32 and BJ cells were kept in DMEM-HG medium, supplemented with $10 \%$ heat-inactivated FBS, 1\% $(v / v)$ penicillin/streptomycin and sodium bicarbonate. The cells were cultured as monolayers and were maintained at $37{ }^{\circ} \mathrm{C}$ in a humidified atmosphere of $5 \% \mathrm{CO}_{2}$.

C32 and BJ cells were seeded in 24-well and 48-well plates at $5 \times 10^{5}$ and $2.5 \times 10^{5}$ cells $/ \mathrm{cm}^{2}$, respectively $(80 \%$ confluence). Plates with a smaller growth area were used for BJ cells, to allow them to better settle down, due to their reduced concentration. The OVPs were added to the wells (in triplicate) $24 \mathrm{~h}$ after plating, to a final concentration ranging from 100 to $1000 \mu \mathrm{M}$. The volume of phenolic solution added to the cultures was adjusted (by changing the concentration of the stock solution) so that the final DMSO concentration in the wells was always kept below the cytotoxic threshold $(0.1 \% v / v)$. Control wells received diluted vehicle only $(0.01 \%(w / v)$ DMSO).

Following a $72 \mathrm{~h}$ incubation period, the growth media was aspirated, the cells were washed, fixed and stored at 20 ${ }^{\circ} \mathrm{C}$. Briefly, fixation was carried out overnight with ice-cold methanol $(1 \%(v / v)$ in acetic acid), after which methanol was decanted and the plate was air-dried.

Cell proliferation was then evaluated through the SRB staining assay to obtain the cellular protein content [50-52]. SRB $(0.5 \%$ in $1 \%$ acetic acid) was added to each well, and the cells were incubated at $37^{\circ} \mathrm{C}$ for 1 hour. Plates were rinsed with $1 \%$ acetic acid, air-dried, and the bound dye

eluted with $1 \mathrm{ml}$ of $10 \mathrm{mM}$ TRIS buffer (pH 10). Absorbance was measured at $540 \mathrm{~nm}$, the amount of dye released being proportional to the number of cells in each well and thus a reliable indicator of cell proliferation.

\section{Statistical Analysis}

All experiments were performed in triplicate. The results were expressed as mean values \pm standard deviation, (the corresponding error bars being displayed in the graphical plots). Statistical analyses were performed using ANOVA, followed by Dunnett post hoc test. Statistical comparison between the data was based on the Pearson correlation coefficient, values less than 0.05 being considered as significant.

\section{RESULTS AND DISCUSSION}

The main phenolic components of virgin olive oil - 3,4DHPEA, OP, 3,4-DHPEA-EA and 3,4-DHPEA-EDA - were screened as to their growth-inhibitory effect towards the C32 human melanoma cell line, and the results interpreted in the light of a previously performed conformational analysis of these compounds by Raman spectroscopy coupled to quantum mechanical calculations (which yielded their most stable structures at physiological conditions) [22]. Actually, it is well recognised that the structural preferences of this type of systems are determinant of their biological function, such as their cytotoxic or cytoprotective activities. In fact, only minor modifications in their structural characteristics (such as the number and position of the ring hydroxyl substituents) are known to drastically affect their bioactivity, similarly to what was verified for other dietary phenols (e.g. hydroxycinnamic and gallic acid derivatives [53-58]).

3,4-DHPEA-EA and 3,4-DHPEA-EDA displayed a clear dose-response inhibition of $\mathrm{C} 32$ cell growth already for the lower concentration tested $(100 \mu \mathrm{M})($ Fig. 3(A) and (B)). In contrast, oleuropein elicited a strong cytoprotective effect

$$
\text { (A) }
$$

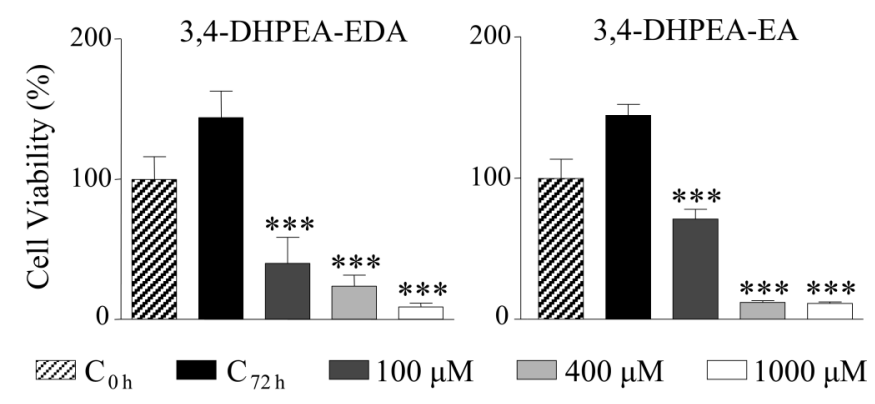

Fig. (3). Dose-dependent antiproliferative effect of 3,4-DHPEA-EA and 3,4-DHPEA-EDA towards the C32 human melanoma cell line, for an incubation time of $72 \mathrm{~h}$. The cell density was assessed by the SRB method (as described in Methods). The results are the mean \pm standard deviation obtained from experiments performed in triplicate, and are expressed as a percentage of the control \pm standard deviation. The one-way ANOVA statistical analysis was used, and the Dunnett's post-test was carried out to verify the significance of the obtained results $(* * * p<0.001$ versus control at $72 \mathrm{~h})$. 


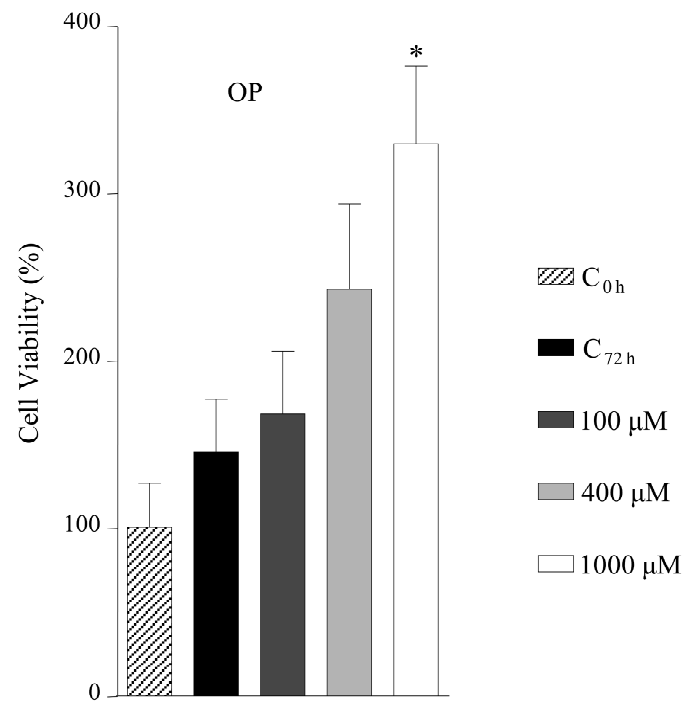

Fig. (4). Dose-dependent cytoprotective effect of OP towards the C32 human melanoma cell line, for an incubation time of $72 \mathrm{~h}$. The cell density was assessed by the SRB method (as described in Methods). The results are the mean \pm standard deviation obtained from experiments performed in triplicate, and are expressed as a percentage of the control \pm standard deviation. The one-way ANOVA statistical analysis was used, and the Dunnett's post-test was carried out to verify the significance of the obtained results $\left({ }^{*} \mathrm{p}<0.05\right.$ versus control at $\left.72 \mathrm{~h}\right)$.

towards this melanoma cell line, at all dosages (Fig. 4). However, Hamdi and Castellon [2] reported this compound to be responsible for a dose-dependent growth inhibition in several human cancer cell lines (namely TF-1a, 786-O, RPMI-7951 and LoVo). Similarly, other studies have found such an antineoplastic effect of oleuropein towards thyroid and breast cancer cells $[11,12,59,60]$, which strongly suggests that the biological activity of this particular phenolic agent may be strongly dependent on the type of cell line (which determines, for instance, the cell uptake process), and urges for further studies in distinct human melanoma cells (e.g. melanotic melanoma). Moreover, the presently measured protective effect of OP on melanoma may be justified by the skin protective action of this phenolic constituent, via its free radical scavenging ability, previously found by Kimura and coworkers [61].

Hydroxytyrosol (3,4-DHPEA), in turn, was found to display a significant anti-proliferative activity towards the melanoma cell line presently studied only for quite high dosages ( $>400 \mu \mathrm{M}$, Fig. 5). Actually, upon a $72 \mathrm{~h}$ incubation with this phenolic compound at $100 \mu \mathrm{M}$ a cytoprotective activity was observed, with a marked increase in C32 cell density (148\%). Regarding non-tumorigenic cells, in turn, hydroxytyrosol was found not to exert this protective effect but a moderate cytotoxic activity instead, for all concentrations tested, as depicted in Fig. 6.

The results currently obtained for 3,4-DHPEA-EDA regarding the melanoma cell line are in accordance with previous data gathered by the authors for colon adenocarcinoma (unpublished data), as well as with reported studies by Fabiani et al. on HL60 leukemia cells [18, 19].

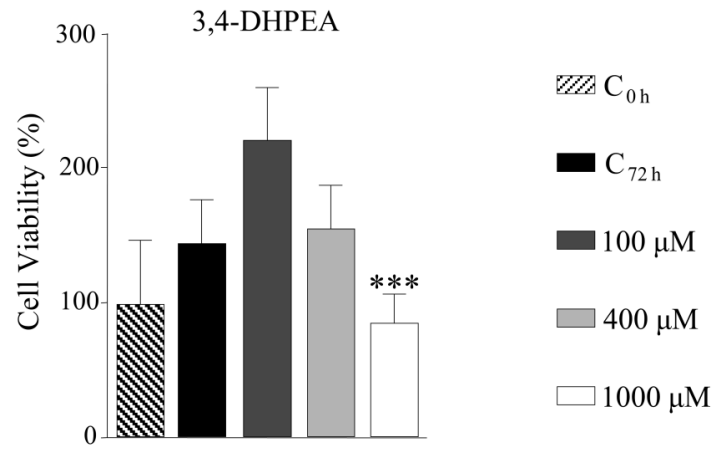

Fig. (5). Dose-dependent antiproliferative effect of 3,4-DHPEA towards the $\mathrm{C} 32$ human melanoma cell line, for an incubation time of $72 \mathrm{~h}$. The cell density was assessed by the SRB method (as described in Methods). The results are the mean \pm standard deviation obtained from experiments performed in triplicate, and are expressed as a percentage of the control \pm standard deviation. The one-way ANOVA statistical analysis was used, and the Dunnett's post-test was carried out to verify the significance of the obtained results $(* * * \mathrm{p}<0.001$ versus control at $72 \mathrm{~h})$.

Regarding the latter, a complete inhibition of growth was reported for a 3,4-DHPEA-EDA concentration equal to 130 $\mu \mathrm{M}$, while a viability decrease to $c a$. $35 \%$ at $100 \mu \mathrm{M}$ was presently observed for the $\mathrm{C} 32$ cells, evidencing a marked selectivity of the compound as regards the type of cancer cell.

The much higher anti-proliferative activity of the conjugated species 3,4-DHPEA-EA and 3,4-DHPEA-EDA is suggested to be related to their distinct structural characteristics as compared to hydroxytyrosol and oleuropein, which establish their lypophilicity versus hydrophilicity and consequently determine their transport processes into the cell and subsequent bioavailability. In fact, linkage of hydroxytyrosol to the di-aldehydic form of elenoic acid involves a drastic change in the physicochemical and structural properties of the resulting conjugates, as was previously shown by the authors through spectroscopic and theoretical methods [22]. The balance between lipo- and hydrophilicity in these systems is of paramount importance for their widespread biodistribution and potential effect within the cellular medium, hydroxytyrosol and oleuropein (comprising a glucose moiety) being significantly more hydrophylic than the corresponding aglycones, which may prevent their prompt cellular uptake. The presence of aldehydic groups in both 3,4-DHPEA-EA and 3,4-DHPEA-EDA, known to be involved in an efficient interaction with proteins (often through lysine residues), may then justify their observed cytotoxicity towards melanoma cells, the compound containg two such moieties displaying a much higher effect at lower dosages $(100 \mu \mathrm{M}$, Fig. 3). Furthermore, the strong lipophilic character of these oil phenolics allows them to interact promptly with the cell membrane, leading to disruption and cell growth impairment, as previously evidenced in red blood cell membranes [62].

Since the concentration of 3,4-DHPEA-EA and 3,4DHPEA-EDA in olive oil is about 10 to 100 times higher than that of the respective alcohols, namely 3,4-DHPEA (although the exact proportion depends on the olive cultivar, 


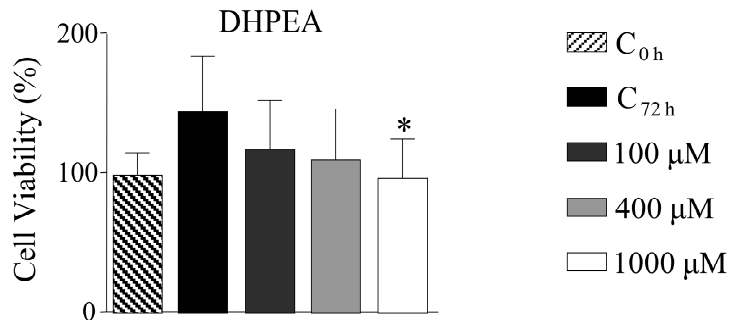

Fig. (6). Dose-dependent antiproliferative effect of 3,4-DHPEA towards the BJ human fibroblast cell line, for an incubation time of $72 \mathrm{~h}$. The cell density was assessed by the SRB method (as described in Methods). The results are the mean \pm standard deviation obtained from experiments performed in triplicate, and are expressed as a percentage of the control \pm standard deviation. The one-way ANOVA statistical analysis was used, and the Dunnett's post-test was carried out to verify the significance of the obtained results $\left({ }^{*} \mathrm{p}<0.05\right.$ versus control at $\left.72 \mathrm{~h}\right)$.

climatic conditions and extraction method), the results presently gathered allow a better understanding, at the molecular level, of the recognised anticancer activity of OVPs.

\section{CONCLUSION}

The main phenolic constituents of virgin olive oil - 3,4DHPEA, OP, 3,4-DHPEA-EDA and 3,4-DHPEA-EA - were assessed as to their in vitro effect on human amelanotic melanoma cells. The aglycones 3,4-DHPEA-EDA and 3,4DHPEA-EA were found to display a significant growthinhibitory activity even at the lowest dosage tested (100 $\mu \mathrm{M})$, as opposed to oleuropein that elicited a strong protective effect at all concentrations $(100-1000 \mu \mathrm{M})$. The smaller oil phenolic 3,4-DHPEA, in turn, led to either a cytoprotective or a cytotoxic effect, in a concentrationdependent way, which was coupled to a modest cytoxicity against non-neoplastic (fibroblast) cells.

This dual effect presently measured for hydroxytyrosol towards amelanotic melanoma cells is in agreement with the known twofold behaviour of phenolic systems, that may act either as antioxidants or pro-oxidants in a concentrationdependent manner [63]. Hence, their overall biological activity is the result of a tight balance between these two abilities, leading to either a cytoprotective or an antiproliferative activity [53]. Moreover, it is expected that in virgin olive oil 3,4-DHPEA, 3,4-DHPEA-EA and 3,4-DHPEAEDA act synergistically with other phenolic components (e.g. lignans), although this has not yet been unequivocally demonstrated and needs further clarification through studies at the molecular level.

According to the recognised structural dependence of the biological activity of phenolic derivatives, namely as chemopreventive agents oxidative-induced carcinogenesis, the previously gathered conformational data on the olive oil constituents presently investigated [22] assisted the interpretation of their biological properties. This type of studies, coupling structural characterisation to biological assessment, allows the establishment of reliable structure activity relationships (SAR's) for natural based polyphenols, ruling their potential cytoprotective role against deleterious oxidative processes vs cytotoxicity towards neoplastic cells [64].

Since unbalanced control of cell growth and differentiation are the main characteristic of neoplasia, natural compounds such as the ones presently studied, consumed in a daily basis (e.g. in a mediterranean diet) and capable of inhibiting cell proliferation, are promising candidates as chemoprotective and/or chemotherapeutic agents. In particular, the knowledge gathered on the interference of this kind of phytochemicals on cell growth may be the basis for the development of topical skin formulations (e.g. for protection against deleterious sunlight UV radiation), as well as for the rational design of functional foods, aiming at cancer prevention (e.g. specifically skin cancer, mainly in populations under increased risk).

\section{CONFLICT OF INTEREST}

The author(s) confirm that this article content has no conflict of interest.

\section{ACKNOWLEDGEMENTS}

The authors acknowledge financial support from the Portuguese Foundation for Science and Technology - UID/ MULTI/00070/2013.

\section{REFERENCES}

[1] Owen, R.W.; Giacosa, A.; Hull, W.E.; Haubner, R.; Wurtele, G.; Spiegelhalder, B.; Bartsch, H. Olive-oil consumption and health: the possible role of antioxidants. Lancet Oncol., 2000, 1(107-112.

[2] Hamdi, H.K.; Castellon, R. Oleuropein, a non-toxic olive iridoid, is an anti-tumor agent and cytoskeleton disruptor. Biochem. Biophys. Res. Commun., 2005, 334(3), 769-778.

[3] Casaburi, I.; Puoci, F.; Chimento, A.; Sirianni, R.; Ruggiero, C.; Avena, P.; Pezzi, V. Potential of olive oil phenols as chemopreventive and therapeutic agents against cancer: a review of in vitro studies. Mol. Nutr. Food Res., 2013, 57(1), 71-83.

[4] Bulotta, S.; Celano, M.; Lepore, S.M.; Montalcini, T.; Pujia, A.; Russo, D. Beneficial effects of the olive oil phenolic components oleuropein and hydroxytyrosol: focus on protection against cardiovascular and metabolic diseases. J. Transl. Med., 2014 12(219), 1-9.

[5] Vissers, M.N.; Zock, P.L.; Katan, M.B. Bioavailability and antioxidant effects of olive oil phenols in humans: a review. Eur. J. Clin. Nutr., 2004, 58(6), 955-965.

[6] Fito, M.; Cladellas, M.; de la Torre, R.; Marti, J.; Alcantara, M.; Pujadas-Bastardes, M.; Marrugat, J.; Bruguera, J.; Lopez-Sabater, M.C.; Vila, J.; Covas, M.I.; members of the, S.I. Antioxidant effect of virgin olive oil in patients with stable coronary heart disease: a randomized, crossover, controlled, clinical trial. Atherosclerosis, 2005, 181(1), 149-158.

[7] Terzuoli, E.; Donnini, S.; Giachetti, A.; Iniguez, M.A.; Fresno, M.; Melillo, G.; Ziche, M. Inhibition of hypoxia inducible factor-1alpha by dihydroxyphenylethanol, a product from olive oil, blocks microsomal prostaglandin-E synthase-1/vascular endothelial growth factor expression and reduces tumor angiogenesis. Clin. Cancer Res., 2010, 16(16), 4207-4216.

[8] Barbaro, B.; Toietta, G.; Maggio, R.; Arciello, M.; Tarocchi, M.; Galli, A.; Balsano, C. Effects of the olive-derived polyphenol oleuropein on human health. Int. J. Mol. Sci., 2014, 15(10), 18508 18524.

[9] Coccia, A.; Mosca, L.; Puca, R.; Mangino, G.; Rossi, A.; Lendaro, E. Extra-virgin olive oil phenols block cell cycle progression and modulate chemotherapeutic toxicity in bladder cancer cells. Oncol. Rep., 2016, 36(6), 3095-3104. 
[10] Terzuoli, E.; Giachetti, A.; Ziche, M.; Donnini, S. Hydroxytyrosol, a product from olive oil, reduces colon cancer growth by enhancing epidermal growth factor receptor degradation. Mol. Nutr. Food Res., 2016, 60(3), 519-529.

[11] Han, J.; Talorete, T.P.; Yamada, P.; Isoda, H. Anti-proliferative and apoptotic effects of oleuropein and hydroxytyrosol on human breast cancer MCF-7 cells. Cytotechnology, 2009, 59(1), 45-53.

[12] Sepporta, M.V.; Fuccelli, R.; Rosignoli, P.; Ricci, G.; Servili, M.; Morozzi, G.; Fabiani, R. Oleuropein inhibits tumour growth and metastases dissemination in ovariectomised nude mice with $\mathrm{MCF}-7$ human breast tumour xenografts. J. Funct. Foods, 2014, 8(2014), 269-273.

[13] Hodge, A.M.; English, D.R.; McCredie, M.R.; Severi, G.; Boyle, P.; Hopper, J.L.; Giles, G.G. Foods, nutrients and prostate cancer. Cancer Causes Control, 2004, 15(1), 11-20.

[14] Fortes, C.; Forastiere, F.; Farchi, S.; Mallone, S.; Trequattrinni, T.; Anatra, F.; Schmid, G.; Perucci, C.A. The protective effect of the Mediterranean diet on lung cancer. Nutr. Cancer, 2003, 46(1), 30-37.

[15] Bosetti, C.; La Vecchia, C.; Talamini, R.; Negri, E.; Levi, F.; Dal Maso, L.; Franceschi, S. Food groups and laryngeal cancer risk: a case-control study from Italy and Switzerland. Int. J. Cancer, 2002, 100(3), 355-360.

[16] Bosetti, C.; Negri, E.; Franceschi, S.; Talamini, R.; Montella, M.; Conti, E.; Lagiou, P.; Parazzini, F.; La Vecchia, C. Olive oil, seed oils and other added fats in relation to ovarian cancer (Italy). Cancer Causes Control, 2002, 13(5), 465-470.

[17] Stoneham, M.; Goldacre, M.; Seagroatt, V.; Gill, L. Olive oil, diet and colorectal cancer: an ecological study and a hypothesis. $J$. Epidemiol. Community Health, 2000, 54(10), 756-760.

[18] Fabiani, R.; De Bartolomeo, A.; Rosignoli, P.; Servili, M.; Montedoro, G.F.; Morozzi, G. Cancer chemoprevention by hydroxytyrosol isolated from virgin olive oil through G1 cell cycle arrest and apoptosis. Eur. J. Cancer Prev., 2002, 11(4), 351-358.

[19] Fabiani, R.; De Bartolomeo, A.; Rosignoli, P.; Servili, M.; Selvaggini, R.; Montedoro, G.F.; Di Saverio, C.; Morozzi, G. Virgin olive oil phenols inhibit proliferation of human promyelocytic leukemia cells (HL60) by inducing apoptosis and differentiation. J. Nutr., 2006, 136(3), 614-619.

[20] Ragione, F.D.; Cucciolla, V.; Borriello, A.; Pietra, V.D.; Pontoni, G.; Racioppi, L.; Manna, C.; Galletti, P.; Zappia, V. Hydroxytyrosol, a natural molecule occurring in olive oil, induces cytochrome c-dependent apoptosis. Biochem. Biophys. Res. Commun., 2000, 278(3), 733-739.

[21] Hashim, Y.Z.; Rowland, I.R.; McGlynn, H.; Servili, M.; Selvaggini, R.; Taticchi, A.; Esposto, S.; Montedoro, G.; Kaisalo, L.; Wahala, K.; Gill, C.I. Inhibitory effects of olive oil phenolics on invasion in human colon adenocarcinoma cells in vitro. Int. J. Cancer, 2008, 122(3), 495-500.

[22] Vissers, M.N.; Zock, P.L.; Roodenburg, A.J.; Leenen, R.; Katan, M.B. Olive oil phenols are absorbed in humans. J. Nutr., 2002, 132(3), 409-417.

[23] Paiva-Martins, F.; Rodrigues, V.; Calheiros, R.; Marques, M.P.M. Characterization of antioxidant olive oil biophenols by spectroscopic methods. J. Sci. Food Agr., 2011, 91(2), 309-314.

[24] Miro-Casas, E.; Covas, M.I.; Farre, M.; Fito, M.; Ortuno, J.; Weinbrenner, T.; Roset, P.; de la Torre, R. Hydroxytyrosol disposition in humans. Clin. Chem., 2003, 49 (6 Pt 1), 945-952.

[25] Weinbrenner, T.; Fito, M.; Farre Albaladejo, M.; Saez, G.T.; Rijken, P.; Tormos, C.; Coolen, S.; De La Torre, R.; Covas, M.I. Bioavailability of phenolic compounds from olive oil and oxidative/antioxidant status at postprandial state in healthy humans. Drugs Exp. Clin. Res., 2004, 30(5-6), 207-212.

[26] Miro Casas, E.; Farre Albadalejo, M.; Covas Planells, M.I.; Fito Colomer, M.; Lamuela Raventos, R.M.; de la Torre Fornell, R. Tyrosol bioavailability in humans after ingestion of virgin olive oil. Clin. Chem., 2001, 47(2), 341-343.

[27] D'Angelo, S.; Manna, C.; Migliardi, V.; Mazzoni, O.; Morrica, P.; Capasso, G.; Pontoni, G.; Galletti, P.; Zappia, V. Pharmacokinetics and metabolism of hydroxytyrosol, a natural antioxidant from olive oil. Drug Metab. Dispos., 2001, 29(11), 1492-1498.

[28] Del Boccio, P.; Di Deo, A.; De Curtis, A.; Celli, N.; Iacoviello, L.; Rotilio, D. Liquid chromatography-tandem mass spectrometry analysis of oleuropein and its metabolite hydroxytyrosol in rat plasma and urine after oral administration. J. Chromatogr. B Analyt. Technol. Biomed. Life Sci., 2003, 785(1), 47-56.
[29] Miro-Casas, E.; Covas, M.I.; Fito, M.; Farre-Albadalejo, M.; Marrugat, J.; de la Torre, R. Tyrosol and hydroxytyrosol are absorbed from moderate and sustained doses of virgin olive oil in humans. Eur. J. Clin. Nutr., 2003, 57(1), 186-190.

[30] Tuck, K.L.; Freeman, M.P.; Hayball, P.J.; Stretch, G.L.; Stupans, I. The in vivo fate of hydroxytyrosol and tyrosol, antioxidant phenolic constituents of olive oil, after intravenous and oral dosing of labeled compounds to rats. J. Nutr., 2001, 131(7), 1993-1996.

[31] Gordon, M.H.; Paiva-Martins, F.; Almeida, M. Antioxidant activity of hydroxytyrosol acetate compared with that of other olive oil polyphenols. J. Agric. Food Chem., 2001, 49(5), 2480-2485.

[32] Paiva-Martins, F.; Gordon, M.H. Effects of $\mathrm{pH}$ and ferric ions on the antioxidant activity of olive polyphenols in oil-in-water emulsions. J. Am. Oil Chem. Soc., 2002, 79(6), 571-576.

[33] Paiva-Martins, F.; Gordon, M.H. Interactions of ferric ions with olive oil phenolic compounds. J. Agric. Food Chem., 2005, 53(7), 2704-2709.

[34] Paiva-Martins, F.; Santos, V.; Mangericao, H.; Gordon, M.H. Effects of copper on the antioxidant activity of olive polyphenols in bulk oil and oil-in-water emulsions. J. Agric. Food Chem., 2006, 54(10), 3738-3743

[35] Visioli, F.; Bellomo, G.; Montedoro, G.; Galli, C. Low density lipoprotein oxidation is inhibited in vitro by olive oil constituents. Atherosclerosis, 1995, 117(1), 25-32.

[36] Fito, M.; Covas, M.I.; Lamuela-Raventos, R.M.; Vila, J.; Torrents, L.; de la Torre, C.; Marrugat, J. Protective effect of olive oil and its phenolic compounds against low density lipoprotein oxidation. Lipids, 2000, 35(6), 633-638.

[37] Covas, M.I.; de la Torre, K.; Farre-Albaladejo, M.; Kaikkonen, J.; Fito, M.; Lopez-Sabater, C.; Pujadas-Bastardes, M.A.; Joglar, J.; Weinbrenner, T.; Lamuela-Raventos, R.M.; de la Torre, R. Postprandial LDL phenolic content and LDL oxidation are modulated by olive oil phenolic compounds in humans. Free Radic. Biol. Med., 2006, 40(4), 608-616.

[38] Manna, C.; Galletti, P.; Cucciolla, V.; Moltedo, O.; Leone, A.; Zappia, V. The protective effect of the olive oil polyphenol $(3,4-$ dihydroxyphenyl)-ethanol counteracts reactive oxygen metaboliteinduced cytotoxicity in Caco-2 cells. J. Nutr., 1997, 127(2), 286292.

[39] D'Angelo, S.; Ingrosso, D.; Migliardi, V.; Sorrentino, A.; Donnarumma, G.; Baroni, A.; Masella, L.; Tufano, M.A.; Zappia, M.; Galletti, P. Hydroxytyrosol, a natural antioxidant from olive oil, prevents protein damage induced by long-wave ultraviolet radiation in melanoma cells. Free Radic. Biol. Med., 2005, 38(7), 908-919.

[40] Quiles, J.L.; Farquharson, A.J.; Simpson, D.K.; Grant, I.; Wahle, K.W. Olive oil phenolics: effects on DNA oxidation and redox enzyme mRNA in prostate cells. Br. J. Nutr., 2002, 88(3), 225-234.

[41] Owen, R.W.; Mier, W.; Giacosa, A.; Hull, W.E.; Spiegelhalder, B.; Bartsch, H. Phenolic compounds and squalene in olive oils: the concentration and antioxidant potential of total phenols, simple phenols, secoiridoids, lignansand squalene. Food Chem. Toxicol., 2000, 38(8), 647-659.

[42] Briante, R.; Febbraio, F.; Nucci, R. Antioxidant properties of low molecular weight phenols present in the mediterranean diet. $J$. Agric. Food Chem., 2003, 51(24), 6975-6981.

[43] Soobrattee, M.A.; Neergheen, V.S.; Luximon-Ramma, A.; Aruoma, O.I.; Bahorun, T. Phenolics as potential antioxidant therapeutic agents: Mechanism and actions. Mutat. Res.-Fund. Mol. M., 2005, 579(1-2), 200-213.

[44] Masella, R.; Cantafora, A.; Modesti, D.; Cardilli, A.; Gennaro, L. Bocca, A.; Coni, E. Antioxidant activity of 3,4-DHPEA-EA and protocatechuic acid: a comparative assessment with other olive oil biophenols. Redox Rep., 1999, 4(3), 113-121.

[45] Manna, C.; D'Angelo, S.; Migliardi, V.; Loffredi, E.; Mazzoni, O. Morrica, P.; Galletti, P.; Zappia, V. Protective effect of the phenolic fraction from virgin olive oils against oxidative stress in human cells. J. Agric. Food Chem., 2002, 50(22), 6521-6526.

[46] Baraldi, P.G.; Simoni, D.; Manfredini, S.; Menziani, E. Preparation of 3,4-Dihydroxy-1-benzeneethanol: A Reinvestigation. Liebigs Ann., 1983, 1983(4), 684-686.

[47] Gariboldi, P.; Jommi, G.; Verotta, L. Secoiridoids from Olea europaea. Phytochemistry, 1986, 25(4), 865-869.

[48] Paiva-Martins, F.; Gordon, M.H. Isolation and characterization of the antioxidant component 3,4-dihydroxyphenylethyl 4-formyl-3- 
formylmethyl-4-hexenoate from olive (Olea europaea) leaves. $J$. Agric. Food Chem., 2001, 49(9), 4214-4219.

[49] Limiroli, R.; Consonni, R.; Ottolina, G.; Marsilio, V.; Bianchi, G.; Zetta, L. 1H and 13C NMR characterization of new oleuropein aglycones. J. Chem. Soc., Perkin Trans. 1, 1995, (12), 1519-1523.

[50] Skehan, P.; Storeng, R.; Scudiero, D.; Monks, A.; McMahon, J.; Vistica, D.; Warren, J.T.; Bokesch, H.; Kenney, S.; Boyd, M.R. New colorimetric cytotoxicity assay for anticancer-drug screening. J. Natl. Cancer Inst., 1990, 82(13), 1107-1112.

[51] Papazisis, K.T.; Geromichalos, G.D.; Dimitriadis, K.A.; Kortsaris, A.H. Optimization of the Sulforhodamine B Colorimetric Assay. $J$. Immunol. Methods 1997, 208(2), 151-158.

[52] Banasiak, D.; Barnetson, A.R.; Odell, R.A.; Mameghan, H.; Russell, P.J. Comparison between the clonogenic, MTT, and SRB assays for determining radiosensitivity in a panel of human bladder cancer cell lines and a ureteral cell line. Radiat. Oncol. Investig., 1999, 7(2), 77-85.

[53] Gomes, C.A.; da Cruz, T.G.; Andrade, J.L.; Milhazes, N.; Borges, F.; Marques, M.P. Anticancer activity of phenolic acids of natural or synthetic origin: a structure-activity study. J. Med. Chem., 2003, 46(25), 5395-5401.

[54] Fiuza, S.M.; Gomes, C.; Teixeira, L.J.; Girao da Cruz, M.T.; Cordeiro, M.N.; Milhazes, N.; Borges, F.; Marques, M.P. Phenolic acid derivatives with potential anticancer properties--a structureactivity relationship study. Part 1: methyl, propyl and octyl esters of caffeic and gallic acids. Bioorg. Med. Chem., 2004, 12(13), 3581-3589.

[55] Teixeira, S.; Siquet, C.; Alves, C.; Boal, I.; Marques, M.P.; Borges, F.; Lima, J.L.; Reis, S. Structure-property studies on the antioxidant activity of flavonoids present in diet. Free Radic. Biol. Med., 2005, 39(8), 1099-1108.

[56] Sousa, J.B.; Calheiros, R.; Rio, V.; Borges, F.; Marques, M.P.M. Conformational analysis of the potential anticancer agent ethyl trihydroxycinnamate - A combined raman spectroscopy and $a b$ initio study. J. Mol. Struct., 2006, 783(1-3), 122-135.

[57] Fresco, P.; Borges, F.; Diniz, C.; Marques, M.P.M. New insights on the anticancer properties of dietary polyphenols. Med. Res. Rev., 2006, 26(6), 747-766.

[58] Machado, N.F.L.; Marques, M.P.M. Bioactive Chromone Derivatives - Structural Diversity Curr. Bioact. Compd., 2010, 6(2), 76-89.

[59] Bulotta, S.; Corradino, R.; Celano, M.; D’Agostino, M.; Maiuolo, J.; Oliverio, M.; Procopio, A.; Iannone, M.; Rotiroti, D.; Russo, D. Antiproliferative and antioxidant effects on breast cancer cells of oleuropein and its semisynthetic peracetylated derivatives. Food Chem., 2011, 127(4), 1609-1614.

[60] Bulotta, S.; Corradino, R.; Celano, M.; Maiuolo, J.; D'Agostino, M.; Oliverio, M.; Procopio, A.; Filetti, S.; Russo, D. Antioxidant and antigrowth action of peracetylated oleuropein in thyroid cancer cells. J. Mol. Endocrinol., 2013, 51(1), 181-189.

[61] Kimura, Y.; Sumiyoshi, M. Olive leaf extract and its main component oleuropein prevent chronic ultraviolet B radiationinduced skin damage and carcinogenesis in hairless mice. J. Nutr., 2009, 139(11), 2079-2086.

[62] Paiva-Martins, F.; Barbosa, S.; Silva, M.; Monteiro, D.; Pinheiro, V.; Mourão, J.L.; Fernandes, J.; Rocha, S.; Belo, L.; Santos-Silva, A. The effect of olive leaf supplementation on the constituents of blood and oxidative stability of red blood cells. J. Funct. Foods 2014, (9), 271-279.

[63] Stevenson, D.E.; Hurst, R.D. Polyphenolic phytochemicals--just antioxidants or much more? Cell. Mol. Life Sci., 2007, 64(22), 2900-2916.

[64] Dias, M.M.; Machado, N.F.L.; Marques, M.P.M. Dietary chromones as antioxidant agents-the structural variable. Food Funct., 2011, 2(10), 595-602. 